\title{
INFLUÊNCIA DO NÚMERO DE NÓS EM ESTACAS SEMILENHOSAS DE OLIVEIRA (Olea europaea L.) NO ENRAIZAMENTO SOB CÂMARA DE NEBULIZAÇÃO ${ }^{1}$
}

\author{
ADELSON FRANCISCO DE OLIVEIRA ${ }^{2}$ \\ MOACIR PASQUAL ${ }^{3}$ \\ NILTON NAGIB JORGE CHALFUN ${ }^{3}$ \\ MURILLO DE ALBUQUERQUE REGINA ${ }^{2}$ \\ CARMEN DEL RIO RINCÓN ${ }^{4}$
}

\begin{abstract}
RESUMO - Com o objetivo de avaliar o enraizamento de estacas semilenhosas de oliveira (Olea europaea L.) com diferentes números de nós, foi conduzido no Centro de Investigación y Formación Agraria - CIFA “Alameda del Obispo" de Córdoba - Espanha, experimento sob condições de câmara de nebulização intermitente. As estacas foram tratadas com ácido indolbutítico (AIB) na concentração de $3.000 \mathrm{mg} . \mathrm{L}^{-1}$, antes da instalação do ensaio, submergindo durante cinco segundos as suas bases, aproximadamente 2,5 centímetros, em solução contendo o produto. Utilizaram-se estacas medianas, que foram coletadas de plantas em desenvolvimento vegetativo contínuo. O delineamento experimental utilizado foi o de blocos casualizados em fatorial $2 \times 5$, sendo respectivamente duas variedades, Picual e Arbequina, e
\end{abstract}

cinco tamanhos de estacas: dois nós com duas folhas, três nós com duas folhas, três nós com quatro folhas, quatro nós com quatro folhas e cinco nós com quatro folhas. Utilizaram-se quatro repetições com parcelas experimentais constituídas por trinta estacas plantadas em substrato de perlita, em mesas suspensas, com aquecimento controlado. Avaliaram-se aos 75 dias porcentagem de estacas enraizadas, mortas e número e comprimento médio de raízes. Observou-se que, entre estacas de quatro nós e cinco nós, com quatro folhas, não houve diferença nos parâmetros considerados; estacas com três nós, com duas e quatro folhas também não diferenciaram. Estacas com dois nós e duas folhas, de ambas as variedades, apresentaram maior mortalidade.

TERMOS PARA INDEXAÇÃO: Olea europaea, propagação, estaquia, tamanho estacas.

\section{INFLUENCE THE NUMBER OF NODES ON SEMI-WOODY CUTTINGS OF OLIVE TREE (Olea europaea L.) ON THE ROOTING IN MISTY ROOM}

\begin{abstract}
With the objective of assessing the rooting efficiency of semi-woody cuttings of olive tree (Olea europaea L.), with different nodes, an experiment under intermittent misty room conditions and with the use of IBA at the concentration of $3000 \mathrm{mg} \mathrm{L}^{-1}$ was run at the Centro de Investigación y Formación Agraria 'Alameda del Obispo' in Cordoba, Spain. The cuttings were treated before the establishment of the trial, soaking their bases, about $2.5 \mathrm{~cm}$, for five minutes in a
\end{abstract}

solution containing the product. Median cuttings, which were collected from plants in continuous vegetative development, were utilized. The experimental design was randomized blocks in a $2 \times 5$ factorial. Two cultivars 'Picual' and 'Arbequina', and five sizes of cuttings: two nodes with two leaves, three nodes with two leaves, three nodes with four leaves, four nodes with four leaves, and five nodes with four leaves were used. Four replicates were

1. Parte da Tese de Doutorado do primeiro autor apresentada à UNIVERSIDADE FEDERAL DE LAVRAS/UFLA Caixa Postal 37 - 37200-000 - Lavras, MG.

2. Pesquisador da Empresa de Pesquisa Agropecuária de Minas Gerais/EPAMIG, Centro Tecnológico Sul de Minas Caixa Postal 176 - 37200.000 - Lavras, MG.

3. Professor do Departamento de Agricultura da UFLA.

4. Pesquisadora do Centro de Investigación y Formación Agraria 'Alameda Del Obispo' de Cordoba, Apartado de Correos 3092, 14080, Cordoba, Espanha. 
utilized, with experimental plots made up of thirty cuttings planted in perlite substrate, on raised tables, with controlled heating. After seventy five days, percentage of roots, dead cuttings, and number and average length of roots were evaluated. It was found that between cuttings of four and five nodes, with four leaves, there were no differences in the considered parameters. Cuttings with three nodes, with two and four leaves also did not distinguish. Cuttings with two nodes and two leaves from both varieties presented greater mortality.

INDEX TERMS: Olea europaea, propagation, cuttings, large cuttings.

\section{INTRODUÇÃO}

A oliveira (Olea europaea L., Oleaceae) é uma arvore de tamanho médio, cujo porte, densidade da copa e cor da madeira variam em função da variedade e de condições de cultivo. Seu sistema radicular está relacionado com origem da árvore; se for de sementes, o sistema radicular é caracterizado por uma raiz pivotante central e a partir de estacas, forma-se um sistema radicular fasciculado. As folhas adultas são simples e de forma elíptica ou lanceolada, com comprimento de 5 a 7 cm e largura de 1,0 a 1,5 cm, sendo a região ventral de cor verde-escura e a região dorsal de cor esbranquiçada devido à presença tricomas ou placas foliares, o que permite resistir às condições de extrema seca. Nos ramos semilenhosos, em cada nó, inserem-se duas folhas opostas, em cujo ponto de inserção encontram-se gemas que, em função do estimulo externo que recebem, podem transformar-se em gemas de brotação ou de florescimento. A flor é constituída por quatro sépalas verdes e por quatro pétalas brancas, que formam a corola. O fruto, denominado azeitona, é uma drupa de tamanho pequeno e forma elipsoidal, cujas dimensões pode apresentar entre 1 a $4 \mathrm{~cm}$ de comprimento e diâmetro de 0,6 a $2 \mathrm{~cm}$ com acumulação de azeite nas células do mesocarpo (Rapoport, 1998).

$\mathrm{Na}$ região mediterrânea, em países da Comunidade Econômica Européia, localizam-se os maiores plantios de oliveira do mundo, onde são produzidos $82 \%$ do azeite de oliva e mais de $90 \%$ de azeitonas para consumo em mesa.

$\mathrm{Na}$ Espanha, na Comunidade Autônoma de Andaluzia, obtêm-se $26 \%$ da produção mundial. Nessa região, o plantio é formado por diferentes variedades, mas com maior importância para 'Picual' e 'Arbequina', cujas principais características são; vigor vegetativo, precocidade, alto rendimento em azeite e boa resistência ao ataque de pragas e doenças.

No Brasil, o consumo de azeitonas e azeite de oliva é suprido com importações, com um gasto anual aproximado de 200 milhões de dólares, principalmente de países como Argentina, Peru e Chile, na América do
Sul. Parte do que se consome no Brasil também é importado da Espanha e Portugal.

Para viabilizar o cultivo econômico dessa planta no Brasil, um aspecto importante é a obtenção de mudas de qualidade.

A oliveira é propagada vegetativamente por meio de rebentos enraizados, óvulos (excrescências lenhosas da base do tronco ricas em gemas adventícias capazes de produzir novos ramos e raízes com alto grau de juvenilidade), enxertia e principalmente por estaquia (Caballero \& del Rio, 1998).

Aproveitar ao máximo a planta matriz fornecedora de material vegetal para a multiplicação da oliveira por estaquia é fundamental para qualquer viveiro de produção de mudas, sobretudo em coleções onde existem poucas árvores de uma mesma variedade ou somente uma, quando esta for obtida de programas de melhoramento genético por cruzamentos interespecíficos.

Para o enraizamento de estacas semilenhosas de oliveira, normalmente essas são utilizadas com cinco nós, no tamanho aproximado de 14 a $16 \mathrm{~cm}$ de comprimento, com 4 ou 6 folhas (Nahlawi et al., 1975; Caballero, 1981; Rkhiss \& Trigui, 1996). Entretanto, seria conveniente diminuir seu tamanho para maximizar o número de plantas possível de obter partindo de um determinado número de ramos. Dessa forma, menos danos seriam causados às plantas-matrizes, sobretudo quando se necessita obter um elevado número de plantas.

Por outro lado, para a produção de assimilados e de outras substâncias necessárias ao enraizamento, a presença de folhas e gemas nas estacas tem um papel-chave para a formação do novo sistema radicular. Foi comprovado que a eliminação de folhas e gemas é prejudicial para a iniciação de raízes e para o crescimento daquelas já formadas (Fontanazza \& Rugini, 1977; Avidan \& Lavee, 1978; Caballero \& Nahlawi, 1979). Nesse sentido, ainda que a diminuição no tamanho da estaca proporcione uma diminuição do número de folhas e de gemas, deve existir um tamanho mínimo que permita seu enraizamento.

De acordo com Hartmann \& Kester (1980), há uma relação entre o enraizamento e o conteúdo de hidratos de carbono presentes nas estacas de algumas es- 
pécies, inclusive para oliveira (Rallo \& del Rio, 1990; del Rio \& Caballero, 1991). Diversos estudos mostram a necessidade de um determinado equilíbrio entre a auxina e hidratos de carbono para a ótima produção de raízes, já que, durante o processo de enraizamento, ocorrem contínuas perdas de amido e açúcares solúveis na base da estaca, que comporta um forte dreno de assimilados (Davis, 1983; del Rio \& Caballero, 1991; Ruiz \& Loreto, 1998).

Estacas de oliveira com diferentes tamanhos, conseqüentemente diferentes conteúdos de hidratos de carbono das cvs. Frantoio e Leccino, postas a enraizar em diversas épocas do ano, apresentaram melhores resultados de enraizamento nos meses de fevereiro-março e agosto-setembro. Neste trabalho foi observado também que, entre estacas com um e dois nós, houve diferença de enraizamento durante todo o período de avaliação (Pannelli et al., 1980).

Dessa forma, realizou-se este trabalho com o objetivo de avaliar a propagação de oliveira por enraizamento de estacas semilenhosas, com diferentes números de nós e folhas, sob condições de câmara de nebulização e com uso de AIB.

\section{MATERIAL E MÉTODOS}

O experimento foi conduzido em casa de nebulização intermitente, com condições de temperatura e umidade controladas $\left(23^{\circ} \mathrm{C}\right.$ e $80 \%$ a $90 \%$ de umidade), do Centro de Investigación y Formación Agraria "Alameda del Obispo" em Córdoba, Espanha, instalado em 05/12/2000.

As estacas foram coletadas de plantas em desenvolvimento vegetativo contínuo das variedades Picual e Arbequina e preparadas com a região mediana do ramo, no mesmo dia da instalação do experimento.

O delineamento experimental utilizado foi o de blocos casualizados em esquema fatorial 2 x 5 , compreendendo duas variedades: Picual e Arbequina, e cinco tamanhos de estacas semilenhosas: dois nós com duas folhas, três nós com duas folhas, três nós com quatro folhas, quatro nós com quatro folhas e cinco nós com quatro folhas, considerado tratamento-testemunha.

As parcelas experimentais, com quatro repetições, foram constituídas por trinta estacas tratadas com o ácido indolbutirico (AIB) na concentração de 3.000 mg. $\mathrm{L}^{-1}$, antes da instalação do ensaio, submergindo a base das respectivas estacas durante cinco segundos (aproximadamente $2,5 \mathrm{~cm}$ ) em solução contendo o produto. Foram plantadas em substrato de perlita em mesas suspensas, com aquecimento controlado, com calor de fundo de 23 a $25^{\circ} \mathrm{C}$.

As avaliações foram realizadas no dia 19/02/2001, sendo consideradas as seguintes características: porcentagem de estacas enraizadas, número e comprimento médio de raízes e porcentagem de estacas mortas.

Os dados coletados, após tabulados, foram analisados estatisticamente pelo Sistema de Análise de Variância para Dados Balanceados (Ferreira, 2000). A comparação das médias foi feita pelo teste Scoott \& Knott (1974), a 5\% de probabilidade.

\section{RESULTADOS E DISCUSSÃO}

Observou-se que a variedade Picual enraizou melhor $(38 \%)$ que Arbequina $(27,50 \%)$ e também apresentou maior número de raízes por estaca $(5,92$ ud para Picual e 4,47 ud para Arbequina, respectivamente). Por outro lado, observou-se um percentual maior de estacas mortas para a variedade Arbequina $(38,33 \%)$, em comparação com a Picual $(29,50 \%)$ (Figura 1).

Não houve diferença estatística entre as médias quando utilizaram-se estacas com quatro nós e quatro folhas $(41,25 \%$ de enraizamento e 6,19 ud de raízes por estaca), comparada com estacas com cinco nós e quatro folhas $(43,75 \%$ de enraizamento e 7,38 ud de raízes por estaca). Também em estacas com três nós a presença de duas folhas a mais não possibilitou melhor enraizamento, pois não foi observada diferença entre médias obtidas para estacas com três nós e duas folhas $(32,50 \%$ de enraizamento e 4,19 ud de raízes por estaca), comparadas com estacas com três nós e quatro folhas $(33,75 \%$ e 4,74 ud) (Figura 1).

Também na Figura 1 observa-se que estacas com dois nós e duas folhas apresentaram menor porcentagem de enraizamento $(12,50 \%)$ e menor número de raízes por estaca (3,48 ud). De acordo com os dados da Figura 1, observa-se uma maior porcentagem de mortalidade quando utilizaram-se estacas com dois nós e duas folhas $(62,08 \%)$. À medida que aumentou seu tamanho, diminuiu o percentual de morte. 

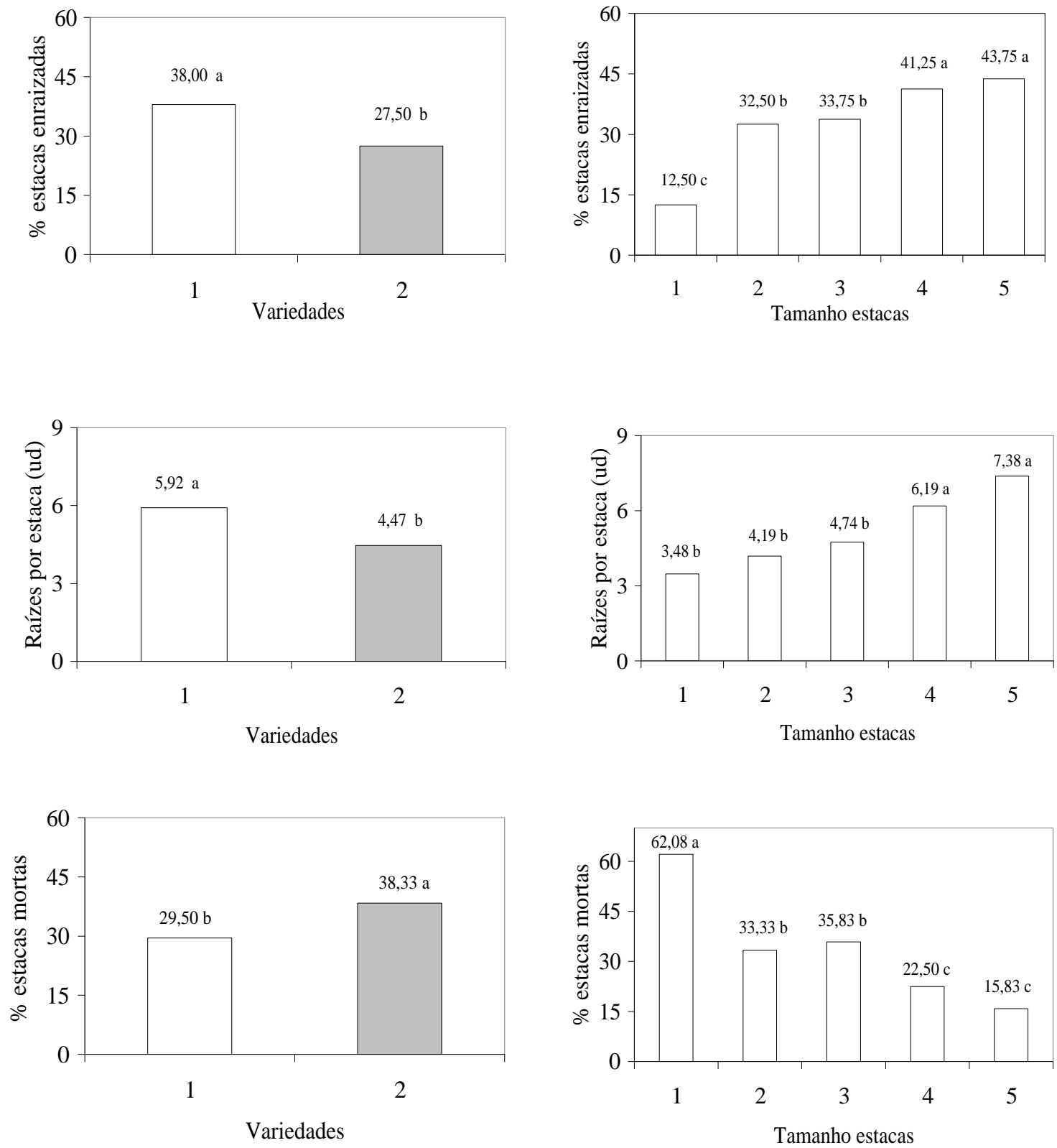

Variedades: 1 - Picual

2 - Arbequina

Tamanho de estacas: 1- (2 nós, 2 folhas); 2- (3 nós, 2 folhas); 3 - (3 nós, 4 folhas); 4- (4 nós, 4 folhas); 5- (5 nós, 4 folhas).

FIGURA 1 - Resultados médios para porcentagem de estacas enraizadas, número de raízes por estaca e porcentagem de estacas mortas, para variedades e tamanho de estacas. Córdoba, Espanha. UFLA, Lavras, MG, 2001.

Na Figura 2 estão os resultados para comprimento médio de raízes, e efeito da interação variedades $\mathrm{x}$ tamanho de estacas.
Para a variedade Picual, observou-se que o comprimento médio de raízes não variou quando utilizaramse estacas com três nós e duas folhas $(3,28 \mathrm{~cm})$, três nós 
e quatro folhas $(3,12 \mathrm{~cm})$, quatro nós e quatro folhas $(3,28 \mathrm{~cm})$ e cinco nós e quatro folhas $(3,91 \mathrm{~cm})$, pois essas médias foram estatisticamente iguais (Figura 2).

Já para variedade Arbequina, observou-se tendência de as estacas maiores apresentarem menor comprimento de raízes, com $2,95 \mathrm{~cm}$ para estacas com cinco nós e quatro folhas, $3,29 \mathrm{~cm}$ para quatro nós e quatro folhas e 3,66 cm para três nós e quatro folhas, embora essas médias tenham sido consideradas estatisticamente iguais (Figura 2).

Por outro lado, tanto para a variedade Picual como para Arbequina, estacas menores apresentaram menores médias de comprimento de raízes: $1,89 \mathrm{~cm}$ para 'Picual', com dois nós e duas folhas, e 1,88 cm e 2,46 cm para 'Arbequina', com dois nós e duas folhas e três nós e duas folhas, respectivamente (Figura 2).

Assim, observou-se que, indiferente do tamanho das estacas utilizadas, a variedade Picual apresentou maior facilidade de emissão de raízes, comparada com Arbequina. Tal resultado já foi comprovado por outros autores, trabalhando com estacas semilenhosas dessa espécie com tamanho padrão, ou seja, cinco nós e quatro folhas, aproximadamente $12 \mathrm{~cm}$ de comprimento (Nahlawi et al., 1975; Caballero, 1981; Rkhiss \& Trigui, 1996).
De acordo com Davis (1983); del Rio \& Caballero (1991) e Ruiz \& Loreto, 1998, durante o enraizamento, a base das estacas apresenta um forte dreno de hidratos de carbono. Dessa forma, o tamanho de estacas reflete a quantidade de reservas a serem utilizadas durante o processo de enraizamento. Estacas pequenas, com dois nós, além de não apresentarem um sistema radicular satisfatório, morreram em maior número do que estacas maiores, com cinco nós.

Em câmara de nebulização, a presença de folhas pode favorecer a emissão de raízes em estacas semilenhosas de oliveira. Isso ocorre porque, durante o processo de enraizamento, é possível que apresente um saldo positivo de hidratos de carbono produzidos pela fotossíntese e utilizados para o enraizamento (Hartmann \& Kester, 1980; Rallo \& del Rio, 1990). Entretanto, no presente experimento, quando utilizaram-se estacas com três nós, não se verificou efeito favorável do número de folhas, pois observou-se, estatisticamente, igual porcentagem de enraizamento entre estacas com duas e quatro folhas. Por outro lado, é importante considerar, nesse caso, que houve uma tendência de estacas com maior número de folhas apresentar maior porcentagem de enraizamento: $33,75 \%$ com quatro folhas e $32,50 \%$ com duas folhas.

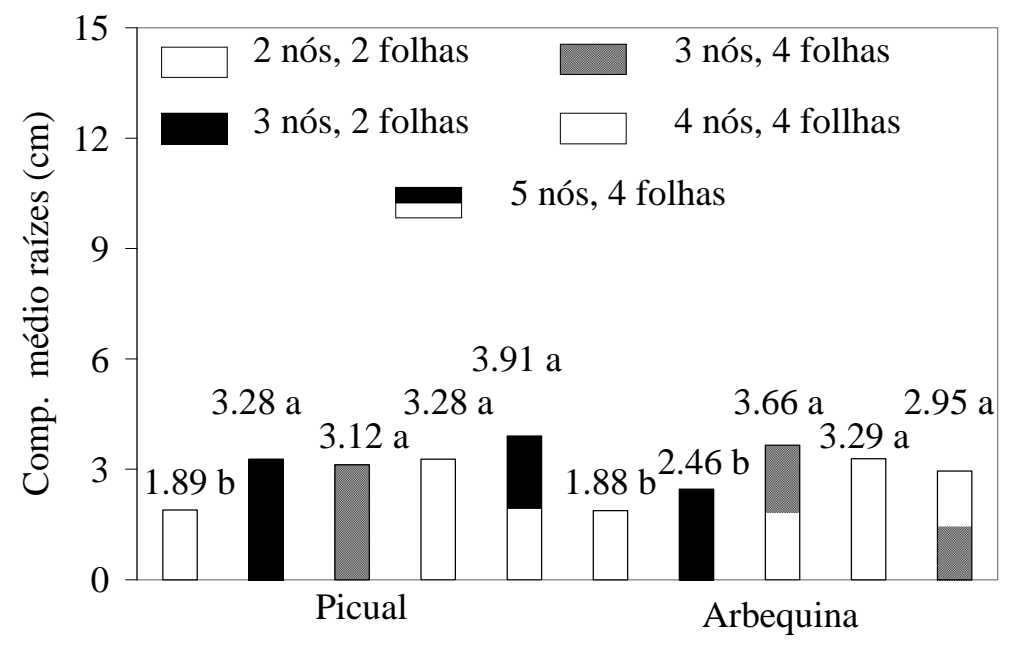

FIGURA 2 - Efeito da interação variedades x tamanho de estacas no comprimento médio de raízes. Córdoba, Espanha. UFLA, Lavras, MG, 2001.

Pannelli et al. (1980), trabalhando com estacas de diferentes tamanhos das cvs. Outros observaram que o enraizamento dessas cultivares apresentam melhores resultados nos meses de fevereiro-março e agosto- setembro. Observaram também que entre estacas com um e dois nós houve diferença de enraizamento durante todo o período de avaliação. 
Resultados semelhantes foram obtidos com o presente trabalho, e estacas com dois e três nós diferiram em todas as características consideradas. Entre estacas com quatro nós e cinco nós e quatro folhas, não foram observadas diferenças estatísticas para os parâmetros considerados.

\section{CONCLUSÕES}

Para as condições em que foi realizado o presente trabalho, conclui-se que:

a) o enraizamento de estacas semilenhosas de oliveira é afetado pelo tamanho das estacas;

b) estacas com tamanhos de quatro nós e quatro folhas e cinco nós e quatro folhas possibilitaram maior porcentagem de enraizamento: $41,25 \%$ e $43,75 \%$;

c) estacas com três nós e duas folhas e três nós e quatro folhas apresentaram porcentagem de enraizamento estatisticamente iguais: $32,50 \%$ e $33,75 \%$;

d) estacas com dois nós e duas folhas apresentaram menor porcentagem de enraizamento $(12,50 \%)$ e maior porcentagem de mortalidade $(62,08 \%)$;

e) para o mesmo tamanho de estacas, a variedade Picual apresentou melhor enraizamento que a variedade Arbequina.

\section{REFERÊNCIAS BIBLIOGRÁFICAS}

AVIDAN, B.; LAVEE, S. Phisiological aspects of the rooting ability of olive cultivars. Acta Horticulturae, Wageningen, v. 79, p. 93-101, 1978.

CABALlERO, J. M. Multiplicación del olivo por etaquillado semileñoso bajo nebulización. Madrid: Instituto Nacional de Investigaciones Agrarias, 1981. 39 p. (Comunicaciones INIA, Serie Producción Vegetal, 31).

CABALlERO, J. M.; del RIO, C. Métodos de multiplicación, 89-113. In: BARRANCO, D. FÉRNANDEZ-ESCOBAR, R.; RALLO, L. (Ed.). El cultivo de olivo. 2. ed. Madri: Junta de Andalucia/Mundi-Prensa, 1998. $651 \mathrm{p}$.

CABALLERO, J. M.; NAHLAWI, N. Influence of carbohydrates and washing with water on the rooting of the Gordal cultivar of the olive (Olea europaea L.). Anais del Instituto Nacional de Investigación Agrarias, Serie ProduciónVegetal, Madrid, v. 11, p. 219-230, 1979.

DAVIS, T. D. Influence of photosyntheseis and carbohidrates on adventitious root formation by leafy cut- tings. Dissertation Abstracts Internacional, Ann Arbor, v. 43, n. 10, p. 3090-3091, 1983.

del RIO, C., CABALlERO, J. M. Effects of carbohydrate content on the seasonal rooting of vegetive and reproductive cuttings of olive. Journal of Horticultural Science, Ashford, v. 66, n. 3, p. 301-309, 1991.

FONTANAZZA, G.; RUGINI, E. Effect of laeves and buds removal on rooting ability of olive tree cuttings. Olea, Cordoba, v. 2, p. 9-28, 1977.

FERREIRA, D. F. Análises estatísticas por meio do Sisvar para Windows versão 4.0. In. REUNIÃO ANUAL DA REGIÃO BRASILEIRA DA SOCIEDADE INTERNACIONAL DE BIOMETRIA. UFSCar, 45., 2000, São Carlos. Anais... São Carlos: SIB, 2000. p. 255-258.

HARTMAnn, H. T.; KeSTER, D. E. Propagacion de plantas, principios y practicas. México: CECSA, 1980. 814 p.

NAHLAWI, N.; RALLO, L. CABALlERO, J. M.; EGUREN, J. The capacity of olive cultivars to root by cuttings under mist [Beta indolebutyric acid, growth substances]. Anais Instituto Nacional de Investigación Agraria, Serie Produción Vegetal, v. 5, p. 167-182, 1975.

PANELLI, G., FILIPUCCI, B.; DADDI, P. Rizogenesi e ciclo vegetativo in Olea europaea L cv. 'Frantoio', 'Leccino' e 'Morailo'. Influenza di trattamenti com fitoreguladori basali e fogliari a diverse concentrazioni. Annali dell'Istituto Sperimentale per l'Olivicoltura, Consenza, v. 6, p. 135-150, 1980.

RALLO, L.; del RIO, C. Effect of a CO2 enriched enviroment on the rooting ability and carbohydrate level of olive cuttings. Advances in Horticultural Science, New York, v. 4, n. 2, p. 129-130, 1990.

RAPOPORT, H. F. Botánica y morfologia. In: BARRANCO, D.; FERNÁNDEZ-ESCOBAR, R.; RALLO, L. El cultivo del olivo. 2. ed. Madri: MundiPrensa- Junta de Andalucia, 1998. 651 p.

RUIZ, G.; LORETO, A. Effect of the application of IBA and date of collection on the rooting of semi-hard cuttings of olive (Olea europaea) cultivar Sevillano. 1998. 53 p. Thesis (Dissertation) - Universidad Catolica de Valparaiso/Faculdade. de Agronomia, Quillota. 
RKHISS, A.; TRIGUI, A. Propagation of SCOTT, A. J.; KNOTT, M. A cluster analysis the "Chemlali de Sfax" by leafy stem cuttings: method for grouping means in the analysis of variconstraints and possibilities of improvement. ance. Biometrics, Washington, v. 30, p. 507-512, Olivae, Madrid, n. 61, p. 46-52; apr. Sept. 1974. 1996. 